\title{
МАТЕМАТИКА
}

\section{О.Л. Бозиев}

\section{РЕШЕНИЕ НЕЛИНЕЙНОГО ГИПЕРБОЛИЧЕСКОГО УРАВНЕНИЯ ПРИБЛИЖЕННО-АНАЛИТИЧЕСКИМ МЕТОДОМ}

\begin{abstract}
Предлагается метод решения смешанной задачи для гиперболического уравнения со степенной нелинейностью путем ее редукции к задаче для нагруженного уравнения, содержащего интеграл натуральной степени модуля неизвестной функции. Последнее уравнение линеаризуется посредством априорных оценок решения поставленной задачи. Получена формула, выражающая его решение через решение обыкновенного дифференциального уравнения, ассоциированного с нагруженным уравнением. Приближение к решению нелинейного уравнения производится с помощью итерационного процесса решения последовательности нелинейных задач.
\end{abstract}

Ключевые слова: нелинейные уравнения в частных производных, нагруженные уравнения в частных производных, априорные оценки, приближенные решения.

Нелинейное уравнение

$$
u_{t t}-a^{2} u_{x x}+b|u|^{p} u_{t}=0
$$

с положительными параметрами $a$ и $b$, натуральным $p$ и начально-краевыми условиями различного вида в прямоугольной области является математической моделью различных нестационарных процессов. В частности, при $p \geq 0$ неоднородное уравнение вида (1) возникает в релятивистской квантовой механике [1, с. 16]. При $p=1$ уравнение (1) моделирует неустановившееся течение жидкости в трубе со скоростью $u(x, t)[2$, с. 42]. Для нахождения приближенного решения (1) с соответствующими условиями, как правило, используются трудоемкие численные методы. В данной статье предлагается приближенно-аналитический метод решения уравнения (1). Для его применения необходимо сначала от (1) перейти к нагруженному [3, с. 17] уравнению

$$
u_{t t}-a^{2} u_{x x}+b u_{t} \int_{\Omega}|u|^{p} d x=0,
$$

которое рассматривается в качестве аппроксимирующего относительно (1) при исходных начальных и граничных условиях. Уравнения вида (2) и его обобщения представляют самостоятельный интерес и исследованы, например, в $[4,5]$, где доказаны теоремы существования и единственности обобщенных решений соответствующих краевых задач. Переход от (1) к (2) позволяет «ослабить» нелинейность исходного уравнения и при этом избежать чрезмерного искажения сути модели- 
руемого процесса. Найденное впоследствии точное или приближенное решение нагруженного уравнения (2) впоследствии можно принять за приближенное решение исходного нелинейного уравнения (1). Такой подход применен в $[6,7]$, где получены формулы общих членов последовательностей приближенных решений начально-краевых задач для некоторых нагруженных уравнений, аппроксимирующих исходные нелинейные уравнения. В [8] для нахождения приближенного решения первой смешанной задачи с однородными граничными условиями для уравнения (2), используются априорные оценки решения поставленной задачи. Ниже используется комбинация этих подходов, в которой для запуска итерационного процесса приближения к регулярному решению задачи (1), (3), (4) предварительно ищется решение задачи (2) - (4) с использованием его же априорных оценок.

\section{1. Априорные оценки}

В области $Q=\{(x, t): 0<x<1,0<t<T\}$ рассмотрим уравнение (2) с натуральной степенью $p \geq 3$. Требуется найти интегрируемую функцию $u(x, t) \in C^{2,2}(\bar{Q})$, удовлетворяющую уравнению (2) в области $Q$, а также условиям

$$
\begin{gathered}
u(x, 0)=0, u_{t}(x, 0)=0,0 \leq x \leq l ; \\
u(0, t)=\psi_{1}(t), u(l, t)=\psi_{2}(t), 0 \leq t \leq T,
\end{gathered}
$$

с функциями $\psi_{1}(t), \psi_{2}(t) \in C^{1}(0, T)$.

Всюду ниже равенство

$$
\|v\|_{p, \Omega}^{p}=\int_{\Omega}|v|^{p} d x
$$

выражает норму функции $v(t)$ в пространстве $L_{p}(\Omega), \Omega=[0, l]$.

Установим некоторые априорные оценки решения задачи (2) - (4), необходимые для нахождения ее приближенного решения.

Сначала умножим уравнение (2) скалярно на функцию $u_{t}$

$$
\left(u_{t t}, u_{t}\right)-a^{2}\left(u_{x x}, u_{t}\right)+b \int_{\Omega}|u|^{p} d x\left(u_{t}, u_{t}\right)=0 .
$$

Преобразуем по отдельности каждое слагаемое:

$$
\begin{gathered}
\left(u_{t t}, u_{t}\right)=\int_{\Omega} u_{t t} u_{t} d x=\frac{1}{2} \int_{\Omega} \frac{\partial}{\partial t}\left(u_{t}\right)^{2} d x=\frac{1}{2} \frac{d}{d t} \int_{\Omega} u_{t}^{2} d x=\frac{1}{2} \frac{d}{d t} \int_{\Omega}\left|u_{t}\right|^{2} d x, \\
-\left(u_{x x}, u_{t}\right)=-\int_{\Omega} u_{x x} u_{t} d x=-\int_{\Omega} \frac{\partial}{\partial x}\left(u_{x} u_{t}\right) d x+\int_{\Omega} u_{x} u_{t x} d x= \\
=-\left.\left(u_{x} u_{t}\right)\right|_{x=0} ^{x=l}+\frac{1}{2} \int_{\Omega} \frac{\partial}{\partial t} u_{x}^{2} d x=u_{x}(0, t) u_{t}(0, t)-u_{x}(l, t) u_{t}(l, t)+\frac{1}{2} \frac{d}{d t} \int_{\Omega} u_{x}^{2} d x= \\
=u_{x}(0, t) \psi_{1 t}(t)-u_{x}(l, t) \Psi_{2 t}(t)+\frac{1}{2} \frac{d}{d t} \int_{\Omega}\left|u_{x}\right|^{2} d x, \\
\left(u_{t}, u_{t}\right)=\int_{\Omega} u_{t}^{2} d x=\int_{\Omega}\left|u_{t}\right|^{2} d x .
\end{gathered}
$$


После подстановки в $\left(2^{\prime}\right)$ приходим к уравнению

$$
\frac{1}{2} \frac{d}{d t} \int_{\Omega}\left(\left|u_{t}\right|^{2}+a^{2}\left|u_{x}\right|^{2}\right) d x+b \int_{\Omega}|u|^{p} d x \int_{\Omega}\left|u_{t}\right|^{2} d x=u_{x}(l, t) \psi_{2 t}(t)-u_{x}(0, t) \psi_{1 t}(t),
$$

интегрируя которое по $t$ в границах от 0 до $t$ получим

$$
\begin{gathered}
\int_{\Omega}\left(\left|u_{t}\right|^{2}+a^{2}\left|u_{x}\right|^{2}\right) d x+2 b \int_{0}^{t} \int_{\Omega}|u|^{p} d x \int_{\Omega}\left|u_{t}\right|^{2} d x d t= \\
=2 \int_{0}^{t}\left(u_{x}(l, t) \psi_{2 t}(t)-u_{x}(0, t) \psi_{1 t}(t)\right) d t+\int_{\Omega}\left(\left|u_{t}(x, 0)\right|^{2}+a^{2}\left|u_{x}(x, 0)\right|^{2}\right) d x .
\end{gathered}
$$

Учитывая начальные условия (3) и неотрицательность второго слагаемого левой части, а также оценивая по модулю первое слагаемое правой части, перейдем от последнего уравнения к неравенствам

$$
\left\|u_{t}\right\|_{2, \Omega}^{2}+a^{2}\left\|u_{x}\right\|_{2, \Omega}^{2} \leq 2 \int_{0}^{t}\left|u_{x}(l, t) \psi_{2 t}(t)+u_{x}(0, t) \psi_{1 t}(t)\right| d t \leq C_{1}(t),
$$

где

$$
\begin{gathered}
C_{1}(t)=2 \max \left\{\max _{t \in[0, T]}\left|u_{x}(l, t)\right|, \max _{t \in[0, T]}\left|u_{x}(0, t)\right|\right\} \int_{0}^{t}\left|\psi_{2 t}(t)+\psi_{1 t}(t)\right| d t=2 C_{0} \int_{0}^{t}\left|\psi_{2 t}(t)+\psi_{1 t}(t)\right| d t, \\
C_{0}=\max \left\{\max _{t \in[0, T]}\left|u_{x}(l, t)\right|, \max _{t \in[0, T]}\left|u_{x}(0, t)\right|\right\} .
\end{gathered}
$$

Отсюда следует, что для всех значений $t \in[0, T]$ выполняются неравенства

$$
\left\|u_{t}\right\|_{2, \Omega}^{2} \leq C_{1}(t),\left\|u_{x}\right\|_{2, \Omega}^{2} \leq \frac{C_{1}(t)}{a^{2}}, C_{1}(0)=0 .
$$

Теорема. Пусть функция $u \in L_{p-2}(\Omega)$ является решением задачи (2) - (4), а неубывающие функции $\psi_{1}(t), \psi_{2}(t) \in L_{p-1}[0, T]$. Тогда функция $\|u\|_{p, \Omega}^{p}$ ограничена константой, зависящей только от $t$.

Доказательство. Умножим уравнение (2) скалярно на функцию $u^{p-1}$

$$
\left(u_{t t}, u^{p-1}\right)-a^{2}\left(u_{x x}, u^{p-1}\right)+b \int_{\Omega}|u|^{p} d x\left(u_{t}, u^{p-1}\right)=0 .
$$

Преобразуем по отдельности каждое слагаемое:

$$
\begin{gathered}
\left(u_{t t}, u^{p-1}\right)=\frac{1}{p} \frac{d^{2}}{d t^{2}} \int_{\Omega} u^{p} d x-(p-1) \int_{\Omega} u_{t}^{2} u^{p-2} d x \\
\left(u_{x x}, u^{p-1}\right)=u_{x}(l, t) \psi_{2}^{p-1}(t)-u_{x}(0, t) \psi_{1}^{p-1}(t)-(p-1) \int_{\Omega} u_{x}^{2} u^{p-2} d x, \\
\int_{\Omega}|u|^{p} d x\left(u_{t}, u^{p-1}\right)=\frac{1}{p} \int_{\Omega}|u|^{p} d x \cdot \frac{d}{d t} \int_{\Omega} u^{p} d x .
\end{gathered}
$$


Вернемся к (6) и умножим его на $\operatorname{sgn}^{p} u$, чтобы перейти к уравнению

$$
\begin{gathered}
\frac{d^{2}}{d t^{2}} \int_{\Omega}|u|^{p} d x+\frac{b}{2} \frac{d}{d t}\left(\int_{\Omega}|u|^{p} d x\right)^{2}=p(p-1) \int_{\Omega}|u|^{p-2}\left(u_{t}^{2}-a^{2} u_{x}^{2}\right) d x+F_{1}(t), \\
F_{1}(t)=p a^{2}\left(u_{x}(l, t) \psi_{2}^{p-1}(t)-u_{x}(0, t) \psi_{1}^{p-1}(t)\right) \operatorname{sgn}^{p} u
\end{gathered}
$$

после интегрирования которого по $t$ с учетом однородности начальных условий получаем

$$
\frac{d}{d t} \int_{\Omega}|u|^{p} d x+\frac{b}{2}\left(\int_{\Omega}|u|^{p} d x\right)^{2}=p(p-1) \int_{0}^{t} \int_{\Omega}|u|^{p-2}\left(u_{t}^{2}-a^{2} u_{x}^{2}\right) d x d t+\int_{0}^{t} F_{1}(t) d t .
$$

К первому слагаемому в правой части (7) применим неравенство Гёльдера:

$$
\int_{0}^{t} \int_{\Omega}|u|^{p-2}\left(u_{t}^{2}-a^{2} u_{x}^{2}\right) d x d t \leq\left(\left.\left.\int_{0}^{t}\left|\int_{\Omega}\right| u\right|^{p-2} d x\right|^{2} d t\right)^{\frac{1}{2}}\left(\int_{0}^{t}\left|\int\right| u_{\Omega}^{2}-a^{2} u_{x}^{2}|d x|^{2} d t\right)^{\frac{1}{2}} .
$$

Сомножители правой части полученного неравенства ограничены: первый в силу $u \in L_{p-2}(\Omega)$ :

$$
\left(\left.\left.\int_{0}^{t}\left|\int_{\Omega}\right| u\right|^{p-2} d x\right|^{2} d t\right)^{\frac{1}{2}} \leq\left(\int_{0}^{t}\left|C_{2}\right|^{2} d t\right)^{\frac{1}{2}}=\sqrt{t}\left|C_{2}\right|,
$$

а второй - в силу оценок (5):

$$
\begin{gathered}
\left(\int_{0}^{t}\left|\int_{\Omega}\right| u_{t}^{2}-a^{2} u_{x}^{2}|d x|^{2} d t\right)^{\frac{1}{2}} \leq\left(\int_{0}^{t}\left|\int_{\Omega}\right| u_{t}^{2}+a^{2} u_{x}^{2}|d x|^{2} d t\right)^{\frac{1}{2}} \leq\left(\int_{0}^{t}\left|\left\|u_{t}\right\|_{2, \Omega}^{2}+a^{2}\left\|u_{x}\right\|_{2, \Omega}^{2}\right|^{2} d t\right)^{\frac{1}{2}} \leq \\
\leq\left(\int_{0}^{t}\left|C_{1}(t)+C_{1}(t)\right|^{2} d t\right)^{\frac{1}{2}}=2\left(\int_{0}^{t}\left|C_{1}(t)\right|^{2} d t\right)^{\frac{1}{2}}=C_{3}(t) .
\end{gathered}
$$

Подставляя сюда $C_{1}(t)$, видим, что

$$
C_{3}(t)=4 C_{0}\left(\int_{0}^{t}\left(\int_{0}^{t}\left|\psi_{2 t}(t)+\psi_{1 t}(t)\right|^{2} d t\right) d t\right)^{\frac{1}{2}} .
$$

Таким образом,

$$
\int_{0}^{t} \int_{\Omega}|u|^{p-2}\left(u_{t}^{2}-a^{2} u_{x}^{2}\right) d x d t \leq \sqrt{t} C_{2} C_{3}(t),
$$

что позволяет перейти от уравнения (7) к неравенству

$$
\frac{d}{d t} \int_{\Omega}|u|^{p} d x+\frac{b}{2}\left(\int_{\Omega}|u|^{p} d x\right)^{2} \leq p(p-1) \sqrt{t} C_{2} C_{3}(t)+\int_{0}^{t}\left|F_{1}(t)\right| d t
$$


Используя свойства функций $\psi_{1}(t)$ и $\psi_{2}(t)$, можно убедиться в том, что

$$
\begin{aligned}
\int_{0}^{t}\left|F_{1}(t)\right| d t & \leq p a^{2}\left(\int_{0}^{t}\left|u_{x}(l, t)\right|\left|\psi_{2}^{p-1}(t)\right| d t+\int_{0}^{t}\left|u_{x}(0, t)\right|\left|\psi_{1}^{p-1}(t)\right| d t\right) \leq \\
& \leq p a^{2}\left(\int_{0}^{t}\left|\psi_{2}(t)\right|^{p-1} d t+\int_{0}^{t}\left|\psi_{1}(t)\right|^{p-1} d t\right) C_{0} .
\end{aligned}
$$

Таким образом, неравенство (8) усилится:

$$
\frac{d}{d t} \int_{\Omega}|u|^{p} d x+\frac{b}{2}\left(\int_{\Omega}|u|^{p} d x\right)^{2} \leq p(p-1) \sqrt{t} C_{2} C_{3}(t)+p a^{2}\left(\int_{0}^{t}\left|\psi_{2}(t)\right|^{p-1} d t+\int_{0}^{t}\left|\psi_{1}(t)\right|^{p-1} d t\right) C_{0} .
$$

Проинтегрируем это соотношение и получим

$$
\int_{\Omega}|u|^{p} d x+\frac{b}{2} \int_{0}^{t}\left(\int_{\Omega}|u|^{p} d x\right)^{2} d t \leq \int_{\Omega}|u(x, 0)|^{p} d x+F(t),
$$

где первое слагаемое правой части равно нулю в силу первого условия (3), а

$$
F(t)=\frac{2}{3} p(p-1) t^{\frac{3}{2}} C_{2} C_{3}(t)+p a^{2}\left(\int_{0}^{t}\left(\int_{0}^{t}\left|\psi_{2}(t)\right|^{p-1} d t+\int_{0}^{t}\left|\psi_{1}(t)\right|^{p-1} d t\right) d t\right) C_{0} t .
$$

Заметим, что $F(t) \leq F(T)$, в силу чего перейдем от (9) к неравенству

$$
\|u\|_{p, \Omega}^{p} \leq \frac{b}{2} \int_{0}^{t}\left(\|u\|_{p, \Omega}^{p}\right)^{2} d t+F(T) .
$$

Применяя к нему следствие из леммы Бихари [9, с.112], получаем оценку

$$
\|u\|_{p, \Omega}^{p} \leq K(t)
$$

с правой частью

$$
K(t)=\frac{2 F(T)}{2-F(T) b t},
$$

выполняющуюся для всех $t \in[0, T], T<2 /(b F(T))$.

Таким образом, теорема доказана.

\section{2. Начальное приближенное решение}

Для нахождения начального приближенного решения задачи (2) - (4) перейдем от (2) к ассоциированному с ним обыкновенному дифференциальному уравнению. Для этого проинтегрируем (2) в границах от 0 до $x$ :

$$
u_{x}(x, t)=\frac{1}{a^{2}} \int_{0}^{x}\left(u_{t t}+b u_{t}\|u\|_{p, \Omega}^{p}\right) d x+A(t) .
$$

Применяя к интегралу теорему о среднем значении, запишем последнее равенство в виде

$$
u_{x}(x, t)=\frac{x}{l a^{2}} \int_{0}^{l}\left(u_{t t}+b u_{t}\|u\|_{p, \Omega}^{p}\right) d x+A(t) .
$$


После повторного интегрирования по $x$ и удовлетворения условий (3) приходим к соотношению

$$
u(x, t)=\frac{x}{2 a^{2}}\left(\frac{x}{l}-1\right)\left(\bar{u}^{\prime \prime}+b \bar{u}^{\prime}\|u\|_{p, \Omega}^{p}\right)+x \frac{\psi_{2}-\psi_{1}}{l}+\psi_{1},
$$

в котором

$$
\bar{u}(t)=\int_{\Omega} u d x .
$$

Применим преобразование (14) к функции (13) для того, чтобы перейти к обыкновенному дифференциальному уравнению

$$
\bar{u}^{\prime \prime}+b\|u\|_{p, \Omega}^{p} \bar{u}^{\prime}+\frac{12 a^{2}}{l^{2}} \bar{u}=\frac{6 a^{2}}{l}\left(\psi_{1}+\psi_{2}\right) .
$$

Начальные условия, необходимые для его интегрирования, получаются из условий (3):

$$
\bar{u}(0)=\int_{\Omega} u(x, 0) d x=0, \bar{u}^{\prime}(0)=\int_{\Omega} u_{t}(x, 0) d x=0 .
$$

Для решения полученной задачи выберем в (11) верхнюю границу неравенства, что позволяет сделать замену

$$
\|u\|_{p, \Omega}^{p}=K(t)
$$

приводящую от (15) к линейному уравнению

$$
\begin{gathered}
\bar{u}^{\prime \prime}+b K(t) \bar{u}^{\prime}+\frac{12 a^{2}}{l^{2}} \bar{u}=F_{2}(t), \\
F_{2}(t)=\frac{6 a^{2}}{l}\left(\psi_{1}+\psi_{2}\right) .
\end{gathered}
$$

Как известно, единственное решение задачи (18), (16) существует для непрерывных функций $K(t)$ и $F_{2}(t)$ при $t \in(0, T)$. После его подстановки вместе с (17) в формулу (13) будет найдена функция

$$
u(x, t)=\frac{3 x}{l}\left(\frac{x}{l}-1\right)\left(\psi_{1}+\psi_{2}-\frac{2}{l} \bar{u}\right)+x \frac{\psi_{2}-\psi_{1}}{l}+\psi_{1},
$$

которую примем за приближенное решение задачи (2) - (4) и начальное приближение аппроксимируемой ею задачи (1), (3), (4).

\section{3. Итерационный процесс}

Функцию (19) примем за нулевое приближение $u^{(0)}$ в итерационном процессе поиска решения задачи (1), (3), (4), состоящем в нахождении «улучшенных» приближенных решений путем последовательного решении задач вида

$$
\begin{gathered}
u_{t t}^{(k)}-a^{2} u_{x x}^{(k)}+b\left|u^{(k-1)}\right|^{p} u_{t}^{(k)}=0 ; \\
u^{(k)}(x, 0)=0, u_{t}^{(k)}(x, 0)=0,0 \leq x \leq l, \\
u^{(k)}(0, t)=\psi_{1}(t), u^{(k)}(l, t)=\psi_{2}(t), 0 \leq t \leq T,
\end{gathered}
$$


где $k=1,2, \ldots-$ итерационный индекс. Процесс завершится при выполнении условия

$$
\left|u^{(k)}(x, t)-u^{(k-1)}(x, t)\right| \leq \varepsilon,
$$

с достаточно малым наперед заданным числом $\varepsilon$ или по достижении заданного количества итераций.

Таким образом, задача решения нелинейного уравнения (1) сводится к последовательному решению линейных уравнений $\left(1^{\prime}\right)$ при соответствующих условиях.

\section{4. Пример}

Абстрагируясь от смысла уравнения (1), примем в нем для упрощения выкладок $a=b=1$. Будем искать решение задачи (2) - (4), рассматривая уравнение (2) в качестве аппроксимирующего для (1).

Пусть $p=3$. Положим $l=1$ и выберем граничные условия (4) в виде $\psi_{1}(t)=$ $=\psi_{2}(t)=1$, тогда в (18) правая часть $F_{2}(t)=12, C_{3}(t)=0$, а величина $F(T)$, определяемая формулой (10), имеет вид

$$
F(T)=3 T^{3} C_{0} .
$$

При этом функция (19) запишется следующим образом:

$$
u(x, t)=6 x(x-1)(1-\bar{u})+1 .
$$

Перейдем к определению постоянных, входящих в эти выражения. Из (12) следует условие положительности функции $K(t)$, а именно, $F(T) t<2$, т.е. $t<2 /\left(3 T^{3} C_{0}\right)$. Так как $t \leq T$, то должно быть $T<2 /\left(3 T^{3} C_{0}\right)$, откуда следует, что $C_{0}<2 /\left(3 T^{4}\right)$. Положим $T=1$, и выберем, например, $C_{0}=0.6$. Затем последовательно найдем $F(T)=1.8, K(t)=1.8 /(1-0.9 t)$. Функция $K(t)$ непрерывна при $t \leq T$. Теперь задача (18), (16) принимает вид

$$
\begin{gathered}
\bar{u}^{\prime \prime}+\frac{1.8}{1-0.9 t} \bar{u}^{\prime}+12 \bar{u}=12, \\
\bar{u}(0)=0, \bar{u}^{\prime}(0)=0 .
\end{gathered}
$$

Ее решением является функция [10, с. 386, ур. 2.104]

$$
\bar{u}(t)=\left(\frac{9}{10} t-1\right) \cos (2 \sqrt{3} t)-\frac{3 \sqrt{3}}{20} \sin (2 \sqrt{3} t)+1,
$$

подстановка которой в (21) дает приближенное решение задачи (2) - (4):

$$
u(x, t)=6 x(x-1)\left(\frac{3 \sqrt{3}}{20} \sin (2 \sqrt{3} t)-\left(\frac{9}{10} t-1\right) \cos (2 \sqrt{3} t)\right)+1 .
$$

Так как уравнение (2) является аппроксимирующим по отношению к уравнению (1) и его интегрирование проводится при тех же условиях (3), (4), что и для уравнения (1), то функцию (22) будем считать начальным приближением решения задачи (1), (3), (4). Чтобы найти «улучшенные» решения, функцию (22), принимаемую за $u^{(0)}$, необходимо подставить в $\left(1^{\prime}\right)$ для запуска итерационного процесса $\left(1^{\prime}\right),\left(3^{\prime}\right),\left(4^{\prime}\right)$. 
На рис. $1-3$ приведены графики функций $u(x, t)$ и $u^{(1)}(x, t)$ при $t=0.4,0.8$ и 1.0 соответственно, полученные путем численного решения задачи (1), (3), (4) и задачи $\left(1^{\prime}\right),\left(3^{\prime}\right),\left(4^{\prime}\right)$ с функцией $u^{(0)}$, определяемой по формуле (21). Сплошная линия соответствует функции $u(x, t)$, а пунктирная $-u^{(1)}(x, t)$.

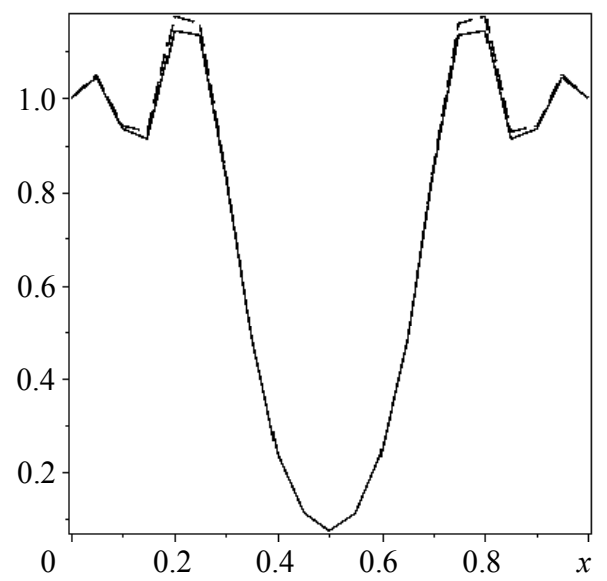

Рис. 1. $t=0.4$

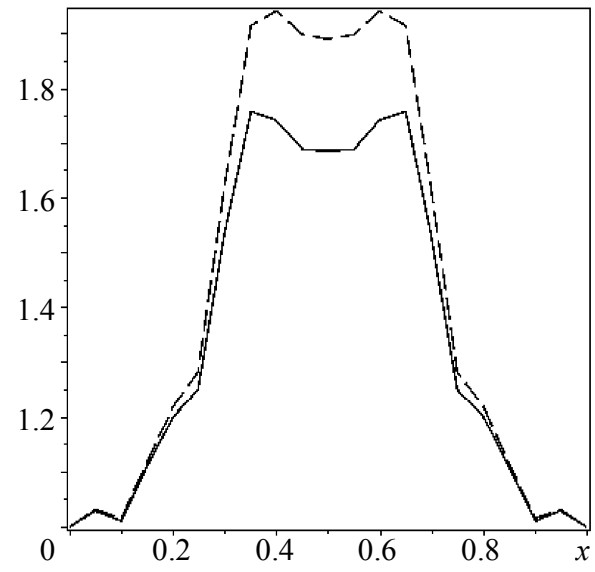

Рис. 2. $t=0.8$

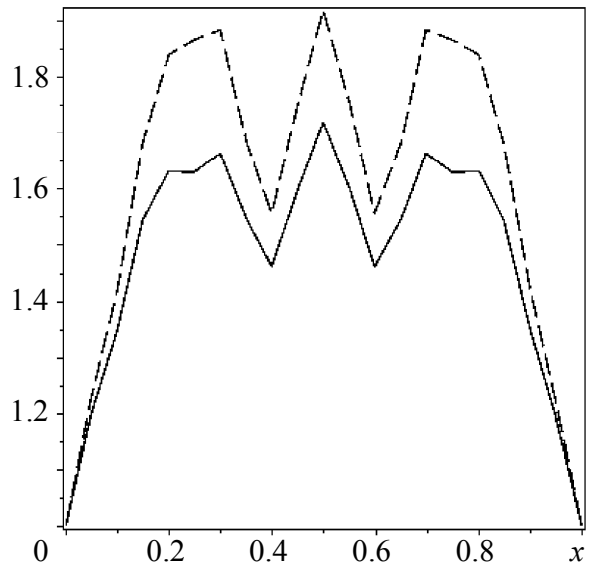

Рис 3. $t=1.0$

Результаты вычислений показывают, что при $t \leq 0.3$ значения этих функций практически совпадают, а их графики не различимы. Увеличение $t$ приводит к росту относительной погрешности вычислений, не превышающей $13.25 \%$. Полученные результаты позволяют предположить, что продолжение итерационного процесса приведет к ее уменьшению.

\section{Заключение}

В работе предложен приближенно-аналитический метод нахождения решения задачи (1), (3), (4), состоящий, во-первых, в переходе от исходного нагруженного уравнения (2) к ассоциированному с ним обыкновенному дифференциальному 
уравнению (15), а во-вторых, в линеаризации (15) с помощью априорной оценки решения исходной задачи вида (11). Полученное начальное приближенное решение выражается аналитически функцией (21). Данная функция используется для запуска итерационного процесса $\left(1^{\prime}\right),\left(3^{\prime}\right),\left(4^{\prime}\right)$, предназначенного для последовательного приближения к (тривиальному) решению задачи (1), (3), (4).

Данный метод применим для приближенного решения дифференциальных уравнений в частных производных со степенной нелинейностью и для решения нагруженных дифференциальных уравнений в частных производных, содержащих интеграл по пространственной переменной от $p$-й степени неизвестной функции при некоторых допущениях относительно $p$. Основную сложность в реализации метода представляют установление априорной оценки вида (11) и подбор констант, входящих в это и другие необходимые неравенства.

\section{ЛИТЕРАТУРА}

1. Лионс Ж.-Л. Некоторые методы решения нелинейных краевых задач. М.: Мир, 1972. $586 \mathrm{c}$.

2. Вишневский К.П. Переходные процессы в напорных системах водоподачи. М.: Агропромиздат, $1986.135 \mathrm{c}$.

3. Нахушев А.М. Нагруженные уравнения и их применение. М.: Наука, 2012. 232 с.

4. Medeiros L. A. On the weak solutions of nonlinear partial differential equations // Anais da Academia Brasileira de Ciencias. 1981. V. 53. No. 1. P. 13-15.

5. Lourêdo A.T., Ferreira de Araújo M.A., Miranda M. M. On a nonlinear wave equation with boundary damping // Mathematical Methods in the Applied Sciences. 2014. V. 37. No. 9. P. 1278-1302.

6. Бозиев О.Л. Решение начально-краевой задачи для нелинейного гиперболического уравнения с помощью двойной редукции к нагруженным уравнениям // Известия Кабардино-Балкарского научного центра РАН. 2014. № 4(60). С. 7-13.

7. Бозиев О.Л. Применение нагруженных уравнений к приближенному решению дифференциальных уравнений в частных производных со степенной нелинейностью // Вестник Тверского государственного университета. Серия: Прикладная математика. 2015. № 1. C. 127-136.

8. Бозиев О.Л. Приближенное решение нагруженного гиперболического уравнения с однородными краевыми условиями // Вестник Южноуральского государственного университета. Серия: Математика, физика, механика. 2016. Т. 8. № 2. С. 14 - 18. DOI: https://doi.org/10.14529/mmph160202.

9. Демидович Б.П. Лекции по математической теории устойчивости. М.: Наука, 1967. $472 \mathrm{c}$.

10. Камке Э. Справочник по обыкновенным дифференциальным уравнениям. М.: Наука, $1971.576 \mathrm{c}$.

Статья поступила 09.06.2017 г.

Boziev O.L. (2018) SOLUTION OF NONLINEAR HYPERBOLIC EQUATIONS BY AN APPROXIMATE ANALYTICAL METHOD. Vestnik Tomskogo gosudarstvennogo universiteta. Matematika i mekhanika [Tomsk State University Journal of Mathematics and Mechanics]. 51. pp. 5-14

\section{DOI $10.17223 / 19988621 / 51 / 1$}

In this paper, we propose a method for solving the mixed problem for a hyperbolic equation with power nonlinearity. The first step of the method is reduction to the problem for the loaded equation containing the integral of a natural degree of the modulus of the unknown function. This integral expresses the norm of the unknown function in the corresponding Lebesgue space. Selection of constants of an a priori estimate allows us to linearize the loaded equation. A formula 
expressing the solution of the loaded equation by the solution of the ordinary differential equation associated with it is obtained. Approximation to the solution of the nonlinear equation is performed by means of an iterative process of solving a sequence of nonlinear problems.

Keywords: nonlinear partial differential equations, loaded partial differential equations, a priori estimates, approximate solutions.

AMS Mathematical Subject Classification: 35L20, 35L72.

BOZIEV Oleg Ludinovich (Candidate of Physics and Mathematics, associate professor of Kabardino-Balkarian State University; senior staff scientist of Institute of Computer Science and Problems of Regional Management of Kabardino-Balkarian Science Center of the Russian Academy of Sciences., Nalchik, Russian Federation)

E-mail: boziev@yandex.ru

\section{REFERENCES}

1. Lions J.L. (1969) Quelques méthods de résolution des problèmes aux limites non linéaire. Paris: Dunod, Gauthier-Villars.

2. Vishnevskii K.P. (1986) Perekhodnye protsessy v napornykh sistemakh vodopodachi [Transient processes in pressurized water systems]. Moscow: Agropromizdat.

3. Nakhushev A.M. (2012) Nagruzhennye uravneniya i ikh primenenie [Loaded equations and their application]. Moscow: Nauka.

4. Medeiros L.A. (1981) On the weak solutions of nonlinear partial differential equations. Anais da Academia Brasileira de Ciências. 53(1). pp. 13-15.

5. Lourêdo A.T., Ferreira de Araújo M.A., Miranda M. M. (2014) On a nonlinear wave equation with boundary damping. Mathematical Methods in the Applied Sciences. 37(9). pp. 12781302.

6. Boziev O.L. (2014) Reshenie nachalno-kraevoy zadachi dlya nelineynogo giperbolicheskogo uravneniya s pomoshch'yu dvoynoy reduktsii k nagruzhennym uravneniyam [Solution of the initially-boundary problem for a nonlinear hyperbolic equation with the use of double reduction to loaded equations]. Izvestiya Kabardino-Balkarskogo nauchnogo tsentra RAN. 4. pp. 7-13.

7. Boziev O.L. (2015) Primenenie nagruzhennyh uravneniy k priblizhennomu resheniyu differentsialnyh uravneniy $\mathrm{v}$ chastnykh proizvodnykh so stepennoy nelineynost'yu [Application of loaded equations to approximate solutions of partial differential equations with the power nonlinearity]. Vestnik TVGU. Ser. Prikl. Matem. - Herald of Tver State University. Ser. Appl. Math. 1. pp. 127-136.

8. Boziev O.L. (2016) Priblizhonnoe reshenie nagrugennogo giperbolicheskogo uravneniya s odnorodnymi kraevymi usloviyami [An approximate solution of loaded hyperbolic equation with homogeneous boundary conditions]. Vestn. Yuzhno-Ural. Gos. Un-ta. Ser. Matem. Mekh. Fiz., 8(2). pp. 14-18. DOI: https://doi.org/10.14529/mmph160202.

9. Demidovich B.P. (1967) Lektsii po matematicheskoy teorii ustoychivosti [Lectures on the mathematical stability theory]. Moscow: Nauka.

10. Kamke E. (1971) Spravochnik po obyknovennym differentsialnym uravneniyam [Differentialgleichungen: Lösungsmethoden und Lösungen]. Moscow: Nauka. 